\title{
LINEAR STABILITY ANALYSIS OF REACTION FRONTS IN LIQUIDS
}

BY

\author{
M. GARBEY, A. TAIK, AND V. VOLPERT
}

Université Lyon 1, France

\begin{abstract}
The propagation of a reaction front for liquid-to-solid reaction is studied. The model includes the heat equation, an equation for the concentration of the liquid reactant, and the equations of liquid motion under the Boussinesq approximation. The linear stability of the reaction front is studied, and conditions for cellular and oscillatory instability are determined.
\end{abstract}

1. Introduction. Experimental observations show that reaction fronts in liquids can cause convective instability [6], [11], [13]-[16], [32]-[34], [37]-[40]. The instability was first demonstrated in the work of Chechilo and Enikolopyan on the propagation of methyl methacrylate polymerization waves [13]-[16]. The physical explanation of this phenomenon is clear: the exothermic reaction leads to a temperature difference near the reaction zone, and convection can occur. Polymerization processes provide interesting examples for the study of the interaction between reaction fronts and thermal and hydrodynamical effects (see [37]).

The articles [13]-[16] led to a systematic experimental and theoretical investigation of frontal polymerization (see [17] and the references cited there), which is a polymerization process where the reaction zone is localized in space. Frontal polymerization has some features in common with condensed-phase combustion; however, the adiabatic heat release and the propagation velocity of the front are usually less for it. Moreover, the reaction kinetics and additional physical effects, such as crystallization, melting, boiling, and convection, in frontal polymerization differ from those in combustion.

The first theoretical investigations of frontal polymerization were concerned with onedimensional models describing the heat diffusion and the kinetics of the process (see [17], [2], [3], [43], [53]-[55]). Later models included effects caused by the change of the viscosity of the medium [8], [12], [45], [59], [60], [42].

A number of articles have been devoted to the study of the multidimensional modes of frontal polymerization which appear as a result of an instability of the uniformly propagating wave. Such propagation modes were first found experimentally [30], [44] and studied theoretically [19], [27], [28], [51], [52] in combustion, and then for polymerization

Received July $15,1993$.

1991 Mathematics Subject Classification. Primary 35K57, 76E15. 
processes [7], [50], [55]. The theoretical analysis of the problem usually involves the investigation of the thermal instability; however, if the reactants are in a liquid phase, the hydrodynamical perturbations can also influence the stability of the front.

In this article we study the interaction between reaction and convection. As mentioned, chemical reactions can lead to convective instability, while hydrodynamical perturbations can cause thermal instability. We perform a linear stability analysis and find the cellular and oscillatory stability boundaries. We note that this problem is related to the interaction between solidification and convection (see [41] and the references cited there). Convection in a porous medium in a chemical reactor was studied in [49]. The articles [36], [47], [48] are devoted to convection caused by a concentration gradient in a reacting medium.

We make the following assumptions in the formulation of the problem.

1. The reagents are in a cylindrical tube, whose axis is in the gravitational force. The tube is long and its radius is large; so we can neglect the influence of the walls.

2. The chemical reaction is a one-step reaction, which is of zero order. The reaction is then given by an expression of the form

$$
W=k(T) \phi(\alpha),
$$

where $T$ is the temperature, $\alpha$ the concentration of the reaction product, and

$$
\phi(\alpha)= \begin{cases}1 & \text { if } \alpha<1 \\ 0 & \text { if } \alpha=1\end{cases}
$$

The temperature dependence of the reaction rate is given by the Arrhenius expression,

$$
k(T)=k_{0} \exp \left(\frac{-E}{R_{0} T}\right),
$$

where $E$ is the activation energy, $R_{0}$ the gas constant, and $k_{0}$ the pre-exponential factor. For the asymptotic analysis of the problem we will assume that the activation energy is large.

We restrict ourselves mostly to the case of ascending front, but will make some remarks for the descending fronts as well.

3. The liquid is incompressible. The density of the medium depends on the temperature only, and the change of the density is small.

4. The coefficient of mass diffusion is much less than the coefficient of heat diffusion, and we can neglect mass diffusion. This assumption is common for frontal polymerization $[17]$.

5. The reaction product is a solid. We assume that the heat release due to solidification or crystallization is much less than the heat release due to the reaction, and that can be neglected entirely.

The contents of the article are as follows. We consider the basic equations in Sec. 2 and reduce the model to a singular perturbation problem in Secs. 3 and 4. The solution of the problem is considered in the form of a perturbed stationary solution. The problem for the perturbations is presented in Sec. 5. The dispersion relation is derived from the solvability condition in Sec. 6. The form of the perturbations is found in Sec. 7. The stability conditions are discussed in Sec. 8. 
2. Basic equations. Under the assumptions above and the Boussinesq approximation we have the following system of equations (see [22]):

$$
\begin{gathered}
\frac{\partial T}{\partial t}+v \nabla T=\kappa \Delta T+q k(T) \phi(\alpha), \\
\frac{\partial \alpha}{\partial t}+v \nabla \alpha=k(T) \phi(\alpha), \\
\frac{\partial v}{\partial t}+(v \nabla) v=-\frac{1}{\rho} \nabla p+\nu \Delta v+g \beta\left(T-T_{0}\right) \gamma, \\
\operatorname{div} v=0 .
\end{gathered}
$$

Here $T$ is the temperature, $\alpha$ the depth of conversion (or the dimensionless concentration of the product of the reaction), $v=\left(v_{x}, v_{y}, v_{z}\right)$ the velocity of the medium, $p$ the pressure, $\kappa$ the coefficient of thermal diffusivity, $q$ the adiabatic heat release, $\rho$ is an average value of the density, $\nu$ the coefficient of the kinematic viscosity, $g$ the gravitational acceleration, $\beta$ the coefficient of thermal expansion, $\gamma$ the unit vector in the $z$-direction (upward),

$$
\nabla=\left(\frac{\partial}{\partial x}, \frac{\partial}{\partial y}, \frac{\partial}{\partial z}\right), \quad \Delta=\frac{\partial^{2}}{\partial x^{2}}+\frac{\partial^{2}}{\partial y^{2}}+\frac{\partial^{2}}{\partial z^{2}}
$$

$x, y, z$ the spatial coordinates, $-\infty<x, y, z<+\infty, t$ the time, $T_{0}$ the mean value of the temperature.

The system (2.1)-(2.4) describes the heat diffusion, chemical reaction, and the motion of an incompressible liquid. The heat released due to the reaction leads to a nonhomogeneous distribution of the temperature, and can cause the convective instability.

If we consider a medium at rest, $v=0$, the system of two equations $(2.1),(2.2)$ describes, in particular, condensed phase combustion [31] or some of the frontal polymerization processes [17]. In this case the stationary propagation of the reaction front (see [35], [23], [46], [56]) and its stability (see [1], [4], [5], [19], [20], [21], [25]) have been studied in detail. In particular, the velocity $c$ of the stationary front propagation and the temperature distribution can be found by an asymptotic analysis for large Zeldovich numbers, $Z=q E /\left(R_{0} T_{b}^{2}\right)[35]$ :

$$
\begin{gathered}
c^{2}=\frac{2 k_{0} \kappa}{q} \frac{R_{0} T_{b}^{2}}{E} \exp \left(\frac{-E}{R_{0} T_{b}}\right), \\
T(\tilde{z}, t)= \begin{cases}T_{b} & \text { if } \tilde{z}<0, \\
T_{i}+\left(T_{b}-T_{i}\right) \exp (-\tilde{z} c / \kappa) & \text { if } \tilde{z}>0,\end{cases} \\
\alpha= \begin{cases}1 & \text { if } \tilde{z}<0, \\
0 & \text { if } \tilde{z}>0 .\end{cases}
\end{gathered}
$$


Here $T_{i}$ is the initial temperature, $T_{i}=\left.T\right|_{z=+\infty}, T_{b}$ is the temperature of the burned mixture, $T_{b}=T_{i}+q, \tilde{z}=z-c t$. This solution is written for the case of the front propagating upward.

We introduce dimensionless spatial variables $x c_{1} / \kappa, y c_{1} / \kappa, z c_{1} / \kappa$, time $t c_{1}^{2} / \kappa$, velocity $c / c_{1}$, and pressure $p /\left(c_{1}^{2} \rho\right)$. Here $c_{1}=c / \sqrt{2}$. Denoting $\theta=\left(T-T_{b}\right) / q$ and keeping for convenience the same notation for other variables, we rewrite the system $(2.1)-(2.4)$ in the form

$$
\begin{aligned}
\frac{\partial \theta}{\partial t}+v \nabla \theta & =\Delta \theta+Z \exp \left(\theta /\left(Z^{-1}+\delta \theta\right)\right) \phi(\alpha), \\
\frac{\partial \alpha}{\partial t}+v \nabla \alpha & =Z \exp \left(\theta /\left(Z^{-1}+\delta \theta\right)\right) \phi(\alpha), \\
\frac{\partial v}{\partial t}+(v \nabla) v & =-\nabla p+P \Delta v+P R\left(\theta+\theta_{0}\right) \gamma,
\end{aligned}
$$

$$
\operatorname{div} v=0
$$

Here $P$ is the Prandtl number, $P=\nu / \kappa, R$ is the Rayleigh number, $R=\left(g \beta q \kappa^{2}\right) /\left(\nu c^{3}\right)$, $\delta=R_{0} T_{b} / E, \theta_{0}=\left(T_{b}-T_{0}\right) / q$.

3. Approximation of infinitely narrow reaction zone. To study the problem analytically we reduce it to a singular perturbation problem where the reaction zone is supposed to be infinitely narrow and the reaction term is neglected outside the reaction zone. This is a common approach for combustion problems [1], [18], [25], [28], [35], [57], [58]. We perform a formal asymptotic analysis with $\varepsilon=Z^{-1}=R_{0} T_{b}^{2} /(q E)$ taken as a small parameter, and obtain a closed interface problem. In this section we do not assume that the product is solid.

We consider a moving frame of reference in which the reaction zone is flat and unmovable. Let $\zeta(x, y, t)$ denote the location of the reaction zone in the laboratory frame of reference. The new independent variable is given by

$$
z_{1}=z-\zeta(x, y, t)
$$

We introduce new functions $\theta_{1}, \alpha_{1}, v_{1}, p_{1}$ :

$$
\begin{array}{ll}
\theta(x, y, z, t)=\theta_{1}\left(x, y, z_{1}, t\right), & \alpha(x, y, z, t)=\alpha_{1}\left(x, y, z_{1}, t\right), \\
v(x, y, z, t)=v_{1}\left(x, y, z_{1}, t\right), & p(x, y, z, t)=p_{1}\left(x, y, z_{1}, t\right) .
\end{array}
$$

We rewrite Eqs. (2.8)-(2.11) in the form (the index 1 for the dependent variables is omitted):

$$
\begin{gathered}
\frac{\partial \theta}{\partial t}-\frac{\partial \theta}{\partial z_{1}} \frac{\partial \zeta}{\partial t}+v \tilde{\nabla} \theta=\tilde{\Delta} \theta+Z \exp \left(\theta /\left(Z^{-1}+\delta \theta\right)\right) \phi(\alpha) \\
\frac{\partial \alpha}{\partial t}-\frac{\partial \alpha}{\partial z_{1}} \frac{\partial \zeta}{\partial t}+v \tilde{\nabla} \alpha=Z \exp \left(\theta /\left(Z^{-1}+\delta \theta\right)\right) \phi(\alpha)
\end{gathered}
$$




$$
\begin{gathered}
\frac{\partial v}{\partial t}-\frac{\partial v}{\partial z_{1}} \frac{\partial \zeta}{\partial t}+(v \tilde{\nabla}) v=-\tilde{\nabla} p+P \tilde{\Delta} v+Q\left(\theta+\theta_{0}\right) \gamma \\
\frac{\partial v_{x}}{\partial x}-\frac{\partial v_{x}}{\partial z_{1}} \frac{\partial \zeta}{\partial x}+\frac{\partial v_{y}}{\partial y}-\frac{\partial v_{y}}{\partial z_{1}} \frac{\partial \zeta}{\partial y}+\frac{\partial v_{z}}{\partial z_{1}}=0
\end{gathered}
$$

where

$$
\begin{gathered}
\tilde{\Delta}=\frac{\partial^{2}}{\partial x^{2}}+\frac{\partial^{2}}{\partial y^{2}}+\frac{\partial^{2}}{\partial z_{1}^{2}}-2 \frac{\partial^{2}}{\partial x \partial z_{1}} \frac{\partial \zeta}{\partial x}-2 \frac{\partial^{2}}{\partial y \partial z_{1}} \frac{\partial \zeta}{\partial y} \\
+\frac{\partial^{2}}{\partial z_{1}^{2}}\left(\left(\frac{\partial \zeta}{\partial x}\right)^{2}+\left(\frac{\partial \zeta}{\partial y}\right)^{2}\right)-\frac{\partial}{\partial z_{1}}\left(\frac{\partial^{2} \zeta}{\partial x^{2}}+\frac{\partial^{2} \zeta}{\partial y^{2}}\right) \\
\tilde{\nabla}=\left(\frac{\partial}{\partial x}-\frac{\partial}{\partial z_{1}} \frac{\partial \zeta}{\partial x}, \frac{\partial}{\partial y}-\frac{\partial}{\partial z_{1}} \frac{\partial \zeta}{\partial y}, \frac{\partial}{\partial z_{1}}\right)
\end{gathered}
$$

We are going to use the method of matched asymptotic expansions. We look for the outer solution of the problem (3.1)-(3.4) in the form of an expansion

$$
\begin{aligned}
& \theta=\theta^{0}+\varepsilon \theta^{1}+\cdots, \quad \alpha=\alpha^{0}+\varepsilon \alpha^{1}+\cdots, \\
& v=v^{0}+\varepsilon v^{1}+\cdots, \quad p=p^{0}+\varepsilon p^{1}+\cdots \text {. }
\end{aligned}
$$

Here $\left(\theta^{0}, \alpha^{0}, v^{0}\right)$ is a dimensionless form of the basic solution given in Sec. 2 .

To obtain the jump conditions in the reaction zone we consider the inner problem. The stretching coordinate is $\eta=z_{1} / \varepsilon, \varepsilon=Z^{-1}$. We look for the inner solution in the form of an expansion

$$
\begin{gathered}
\theta=\varepsilon \tilde{\theta}^{1}+\cdots, \quad \alpha=\tilde{\alpha}^{0}+\varepsilon \tilde{\alpha}^{1}+\cdots \\
v=\tilde{v}^{0}+\varepsilon \tilde{v}^{1}+\cdots, \quad p=\tilde{p}^{0}+\varepsilon \tilde{p}^{1}+\cdots, \quad \zeta=\zeta^{0}+\varepsilon \zeta^{1}+\cdots
\end{gathered}
$$

Substituting these expansions into (3.1)-(3.4), we obtain the first-order inner problem:

$$
\begin{gathered}
\left(1+\left(\frac{\partial \zeta^{0}}{\partial x}\right)^{2}+\left(\frac{\partial \zeta^{0}}{\partial y}\right)^{2}\right) \frac{\partial^{2} \tilde{\theta}^{1}}{\partial \eta^{2}}+\exp \left(\tilde{\theta}^{1} /\left(1+\delta \tilde{\theta}^{1}\right) \phi\left(\tilde{\alpha}^{0}\right)=0\right. \\
-\frac{\partial \tilde{\alpha}^{0}}{\partial \eta} \frac{\partial \zeta^{0}}{\partial t}-\frac{\partial \tilde{\alpha}^{0}}{\partial \eta}\left(\tilde{v}_{x}^{0} \frac{\partial \zeta^{0}}{\partial x}+\tilde{v}_{y}^{0} \frac{\partial \zeta^{0}}{\partial y}-\tilde{v}_{\eta}^{0}\right)=\exp \left(\tilde{\theta}^{1} /\left(1+\delta \tilde{\theta}^{1}\right)\right) \phi\left(\tilde{\alpha}^{0}\right) \\
\left(1+\left(\frac{\partial \zeta^{0}}{\partial x}\right)^{2}+\left(\frac{\partial \zeta^{0}}{\partial y}\right)^{2}\right) \frac{\partial^{2} \tilde{v}^{0}}{\partial \eta^{2}}=0
\end{gathered}
$$




$$
-\frac{\partial \tilde{v}_{x}^{0}}{\partial \eta} \frac{\partial \zeta^{0}}{\partial x}-\frac{\partial \tilde{v}_{y}^{0}}{\partial \eta} \frac{\partial \zeta^{0}}{\partial y}+\frac{\partial \tilde{v}_{\eta}^{0}}{\partial \eta}=0
$$

The matching conditions are

$$
\begin{gathered}
\eta \rightarrow \infty:\left.\quad \tilde{\theta}^{1} \sim \theta^{1}\right|_{z_{1}=+0}+\left(\left.\frac{\partial \theta^{0}}{\partial z_{1}}\right|_{z_{1}=+0}\right) \eta, \quad \tilde{\alpha}^{0} \rightarrow 0,\left.\quad \tilde{v}^{0} \rightarrow v^{0}\right|_{z_{1}=+0}, \\
\eta \rightarrow-\infty:\left.\quad \tilde{\theta}^{1} \sim \theta^{1}\right|_{z_{1}=-0}, \quad \tilde{\alpha}^{0} \rightarrow 1,\left.\quad \tilde{v}^{0} \rightarrow v^{0}\right|_{\tilde{z}_{1}=-0} .
\end{gathered}
$$

From (3.9) and the boundedness of the solution we conclude that $\tilde{v}^{0}$ does not depend on $\eta$. From this conclusion and the matching conditions it follows that the first element of the expansion of the velocity for the outer problem, $v^{0}$, is continuous. We denote

$$
s=\tilde{v}_{x}^{0} \frac{\partial \zeta^{0}}{\partial x}+\tilde{v}_{y}^{0} \frac{\partial \zeta^{0}}{\partial y}-\tilde{v}_{\eta}^{0}
$$

From (3.10) it follows that the function $s$ does not depend on $\eta$.

We derive next the jump conditions for the temperature from (3.7), (3.8) in the same way as is usually done for the combustion problems [1], [4], [25], [26], [28]. From (3.8) it follows that $\tilde{\alpha}^{(0}$ is a monotone function and $0<\tilde{\alpha}^{0}<1$. Since we consider zero-order reaction, we have $\phi\left(\tilde{\alpha}^{0}\right) \equiv 1$. We conclude from (3.7) that $\tilde{\theta}^{1}$ is also monotone. Thus, multiplying (3.7) by $\frac{\partial \tilde{\theta}^{1}}{\partial \eta}$ and integrating, we obtain

$$
\left.\left(\frac{\partial \tilde{\theta}^{1}}{\partial \eta}\right)^{2}\right|_{\infty}-\left.\left(\frac{\partial \tilde{\theta}^{1}}{\partial \eta}\right)^{2}\right|_{-\infty}=2 A^{-1} \int_{-\infty}^{\theta^{1}} \exp (\tau /(1+\delta \tau)) d \tau
$$

where

$$
A=1+\left(\frac{\partial \zeta^{0}}{\partial x}\right)^{2}+\left(\frac{\partial \zeta^{0}}{\partial y}\right)^{2}
$$

Subtracting (3.7) from (3.8) and integrating, we have

$$
\left.\frac{\partial \tilde{\theta}^{1}}{\partial \eta}\right|_{\infty}-\left.\frac{\partial \tilde{\theta}^{1}}{\partial \eta}\right|_{-\infty}=-A^{-1}\left(\frac{\partial \zeta^{()}}{\partial t}+s\right)
$$

Using the matching conditions and truncating the expansion (see, for example, [25]):

$$
\theta^{0} \approx \theta,\left.\left.\quad \theta^{1}\right|_{z_{1}=-0} \approx Z \theta\right|_{z_{1}=0}, \quad \zeta^{0} \approx \zeta, \quad v^{0} \approx v
$$


we obtain the jump conditions

$$
\begin{aligned}
\left.\left(\frac{\partial \theta}{\partial z_{1}}\right)^{2}\right|_{+0} & -\left.\left(\frac{\partial \theta}{\partial z_{1}}\right)^{2}\right|_{-0} \\
& =2 Z\left(1+\left(\frac{\partial \zeta}{\partial x}\right)^{2}+\left(\frac{\partial \zeta}{\partial y}\right)^{2}\right)^{-1} \int_{-\infty}^{\left.\theta\right|_{0}} \exp \left(\tau /\left(Z^{-1}+\delta \tau\right)\right) d \tau
\end{aligned}
$$

4. Formulation of the problem. We recall that the jump conditions (3.13), (3.14) have been derived in the general case. In this section we consider the case of the solid product where the velocity is zero behind the reaction zone, $v \equiv 0$ for $z<\zeta$. Thus we have the following formulation of the problem:

$z>\zeta$ :

$$
\begin{gathered}
\frac{\partial \theta}{\partial t}+v \nabla \theta=\Delta \theta \\
\alpha=0, \\
\frac{\partial v}{\partial t}+(v \nabla) v=-\nabla p+P \Delta v+Q\left(\theta+\theta_{0}\right) \gamma, \\
\operatorname{div} v=0 ;
\end{gathered}
$$

$z<\zeta:$

$$
\begin{gathered}
\frac{\partial \theta}{\partial t}=\Delta \theta \\
\alpha=1, \quad v=0 ;
\end{gathered}
$$

$z=\zeta$ :

$$
\left.\theta\right|_{\zeta-0}=\left.\theta\right|_{\zeta+0},
$$

$$
\left.\frac{\partial \theta}{\partial z}\right|_{\zeta-0}-\left.\frac{\partial \theta}{\partial z}\right|_{\zeta+0}=\left(1+\left(\frac{\partial \zeta}{\partial x}\right)^{2}+\left(\frac{\partial \zeta}{\partial y}\right)^{2}\right)^{-1} \frac{\partial \zeta}{\partial t}
$$

$\left.\left(\frac{\partial \theta}{\partial z}\right)^{2}\right|_{\zeta-0}-\left.\left(\frac{\partial \theta}{\partial z}\right)^{2}\right|_{\zeta+0}=-2 Z\left(1+\left(\frac{\partial \zeta}{\partial x}\right)^{2}+\left(\frac{\partial \zeta}{\partial y}\right)^{2}\right)^{-1} \int_{-\infty}^{\left.\theta\right|_{\zeta}} \exp \left(\tau /\left(Z^{-1}+\delta \tau\right)\right) d \tau$

$$
v_{x}=v_{y}=v_{z}=0 .
$$


The conditions at infinity are

$$
z=-\infty: \quad \theta=0, \quad z=+\infty: \quad \theta=-1, v=0 .
$$

The problem (4.1)-(4.11) is coupled in the sense that it describes the thermal instability of the reaction front and the convective instability at the same time. There are different limiting cases here. For example, if the coefficient of the thermal expansion, $\beta$, is zero (i.e., $R=0$ ) then we have, obviously, a pure problem of the reaction in a condensed medium, since $v \equiv 0$. Another limiting case is when we remove the thermal instability: we will see further that for the cellular instability the perturbation of temperature is zero for $z \leq \zeta$. Thus, it can be treated as purely convective instability.

5. Linear stability. The problem (4.1)-(4.11) has a travelling wave solution:

$$
\theta(x, y, z, t)=\theta_{s}(z-u t), \quad \alpha(x, y, z, t)=\alpha_{s}(z-u t), \quad v=0,
$$

which coincides with the basic solution given above:

$$
\theta_{s}\left(z_{2}, t\right)=\left\{\begin{array}{ll}
0 & z_{2}<0 \\
\exp \left(-u z_{2}\right)-1 & z_{2}>0
\end{array}, \quad \alpha_{s}\left(z_{2}, t\right)= \begin{cases}1 & z_{2}<0 \\
0 & z_{2}>0\end{cases}\right.
$$

$z_{2}=z-u t, u=c$. This is a stationary solution of the problem given by Eqs. (4.2), (4.4), $(4.6)-(4.11)$, and

$$
\begin{gathered}
\frac{\partial \theta}{\partial t}+v \nabla \theta=\Delta \theta+u \frac{\partial \theta}{\partial z_{2}} \\
\frac{\partial v}{\partial t}+(v \nabla) v=-\nabla p+P \Delta v+u \frac{\partial v}{\partial z_{2}}+Q\left(\theta+\theta_{0}\right) \gamma
\end{gathered}
$$

for $z_{2}>\xi$

$$
\frac{\partial \theta}{\partial t}=\Delta \theta+u \frac{\partial \theta}{\partial z_{2}}
$$

for $z_{2}<\xi$. Here $\xi=\zeta-u t$.

We study the linear stability of this solution by looking for a solution of the problem in the form of the perturbed stationary solution:

$$
\theta=\theta_{s}+\tilde{\theta}, \quad v=v_{s}+\tilde{v}, \quad p=p_{s}+\tilde{p} .
$$

We substitute (5.1) into (4.4), (5.2) (5.4) and obtain for the first-order terms:

$$
z_{2}>\xi:
$$

$$
\begin{gathered}
\frac{\partial \tilde{\theta}}{\partial t}=\Delta \tilde{\theta}+u \frac{\partial \tilde{\theta}}{\partial z_{2}}-\tilde{v}_{z} \theta_{s}^{\prime} \\
\frac{\partial \tilde{v}}{\partial t}=-\nabla \tilde{p}+P \Delta \tilde{v}+u \frac{\partial \tilde{v}}{\partial z_{2}}+Q \tilde{\theta} \gamma \\
\operatorname{div} \tilde{v}=0
\end{gathered}
$$

$z_{2}<\xi$

$$
\frac{\partial \tilde{\theta}}{\partial t}=\Delta \tilde{\theta}+u \frac{\partial \tilde{\theta}}{\partial z_{2}}
$$


We denote $\tilde{\theta}=\hat{\theta}_{1}$ for $z_{2}<\xi$ and $\tilde{\theta}=\hat{\theta}_{2}$ for $z_{2}>\xi$.

We linearize now the jump conditions (4.7)-(4.10). Taking into account that

$$
\begin{gathered}
\left.\theta\right|_{\xi \pm 0}=\theta_{s}(0)+\xi \theta_{s}^{\prime}( \pm 0)+\tilde{\theta}( \pm 0), \\
\left.\frac{\partial \theta}{\partial z_{2}}\right|_{\xi \pm 0}=\theta_{s}^{\prime}( \pm 0)+\xi \theta_{s}^{\prime \prime}( \pm 0)+\left.\frac{\partial \tilde{\theta}}{\partial z_{2}}\right|_{\xi \pm 0}
\end{gathered}
$$

up to the higher-order terms, we obtain

$$
\begin{gathered}
{[\hat{\theta}]=u \xi,} \\
{\left[\hat{\theta}^{\prime}\right]=-u^{2} \xi-\xi^{\prime},} \\
-u\left(u^{2} \xi+\hat{\theta}_{2}^{\prime}(0)\right)=Z \hat{\theta}_{1}(0) .
\end{gathered}
$$

Here

$$
[\hat{\theta}]=\hat{\theta}_{2}(0)-\hat{\theta}_{1}(0), \quad\left[\hat{\theta}^{\prime}\right]=\hat{\theta}_{2}^{\prime}(0)-\hat{\theta}_{1}^{\prime}(0), \quad \hat{\theta}_{i}^{\prime}(0)=\left.\frac{\partial \hat{\theta}_{i}}{\partial z_{2}}\right|_{z_{2}=0}, \quad \xi^{\prime}=\frac{d \xi}{d t}
$$

From (4.10) we have

$$
\frac{\partial v_{x}}{\partial x}+\frac{\partial v_{x}}{\partial z} \frac{\partial \xi}{\partial x}=0, \quad \frac{\partial v_{y}}{\partial y}+\frac{\partial v_{y}}{\partial z} \frac{\partial \xi}{\partial y}=0
$$

Thus, using (4.4), we can rewrite the boundary conditions (4.10) for the first-order terms in the form

$$
\tilde{v}_{z}=0, \quad \frac{\partial \tilde{v}_{z}}{\partial z}=0
$$

As usual, the solvability conditions of the system (5.6)-(5.9) will give the dispersion relation.

6. Dispersion relation. We consider perturbations of the form

$$
\begin{aligned}
\hat{\theta}_{i} & =\theta_{i}\left(z_{2}\right) \exp \left(\omega t+i\left(k_{1} x+k_{2} y\right)\right), \quad i=1,2, \\
\tilde{v} & =v_{2}\left(z_{2}\right) \exp \left(\omega t+i\left(k_{1} x+k_{2} y\right)\right), \quad z_{2}>\xi \\
\xi & =\varepsilon_{1} \exp \left(\omega t+i\left(k_{1} x+k_{2} y\right)\right) .
\end{aligned}
$$

Here $\varepsilon_{1}$ is the amplitude, $\omega$ the frequency, $k_{1}$ and $k_{2}$ the wave numbers.

From (5.5)-(5.8) we obtain

$$
\begin{gathered}
{[\theta]=\varepsilon_{1} u,} \\
{\left[\theta^{\prime}\right]=-\varepsilon_{1}\left(\omega+u^{2}\right),} \\
-u\left(u^{2} \varepsilon_{1}+\theta_{2}^{\prime}\right)=Z \theta_{1}(0),
\end{gathered}
$$


and

$$
\theta_{1}^{\prime \prime}+u \theta_{1}^{\prime}-\left(\omega+k^{2}\right) \theta_{1}=0
$$

$k^{2}=k_{1}^{2}+k_{2}^{2}$. Thus

$$
\theta_{1}=c_{1} e^{\mu_{1} z_{2}}, \quad \mu_{1}=\frac{u}{2}(-1+d), \quad d=\sqrt{1+\frac{4 \omega}{u^{2}}+\frac{4 k^{2}}{u^{2}}} .
$$

We derive the dispersion relation as the solvability condition of (6.4)-(6.6). If $c_{1}=0$, then from (6.5), (6.6) we conclude that $\omega=0$. This case is considered below as a particular case of the general dispersion relation. We assume now that $c_{1} \neq 0$. Then

$$
\begin{gathered}
-u \frac{\theta_{1}^{\prime}(0)}{\theta_{1}(0)}+\omega\left(\frac{\theta_{2}(0)}{\theta_{1}(0)}-1\right)=Z, \\
\frac{\theta_{1}^{\prime}(0)}{\theta_{1}(0)}-\frac{\theta_{2}^{\prime}(0)}{\theta_{1}(0)}=\frac{\omega+u^{2}}{u}\left(\frac{\theta_{2}(0)}{\theta_{1}(0)}-1\right) .
\end{gathered}
$$

If $\theta_{2}(0)=0$, then from $(6.8)$

$$
Z=-\omega+u^{2} \frac{1-d}{2} .
$$

It is easy to verify that this equation does not have a solution for $\omega=i \phi, \phi \neq 0$. This means that, for the oscillatory stability boundary, $\theta_{2}(0) \neq 0$. In this case we have from $(6.8),(6.9)$

$$
-\sigma\left(\omega_{1}+Z_{1}+\frac{d-1}{2}\right)=Z_{1}+\frac{d-1}{2}+\omega_{1} Z_{1},
$$

where $\omega_{1}=\omega / u^{2}, Z_{1}=Z / u^{2}$,

$$
\sigma=\frac{1}{u} \frac{\theta_{2}^{\prime}(0)}{\theta_{2}(0)} .
$$

We note that the dispersion relation (6.10) is derived from the matching condition only, and in this sense it has a general form. Later in this section we consider general properties of the dispersion relation. For a concrete problem $\sigma$ can be found as a function of parameters from the equations for perturbations. We find this function $\sigma=\sigma(R, P, k, u, \omega)$ in the next section, and then obtain the stability conditions.

We consider some particular cases of the general dispersion relation (6.10).

1. If $\omega=0$ then from (6.10) we obtain the equality

$$
\sigma(R, P, k, u, 0)=-1,
$$

which gives the cellular stability boundary.

We note that in this case $c_{1}=0$. Indeed, otherwise we obtain from (6.5), (6.6) the equality

$$
Z=\frac{u^{2}}{2}(1-d)
$$

which cannot take place since $Z>0, d \geq 1$. Thus the perturbation of the temperature behind the reaction front is zero, and the temperature is equal to the burning temperature. 
2. If the Rayleigh number is zero $(Q=0)$ then the perturbation of the velocity is zero for $z_{2}>\xi$. In this case we have the combustion problem and

$$
\theta_{2}=c_{2} e^{\mu_{2} z_{2}}, \quad \mu_{2}=-\frac{u}{2}(1+d)
$$

Thus

$$
\sigma=-\frac{1+d}{2}
$$

The dispersion relation (6.10) coincides in this case with that for the condensed phase combustion [1]:

$$
2 Z_{1}\left(2 \omega_{1}+1-d\right)=2 \omega_{1}(1+d)+(1-d)^{2}
$$

3. We consider now a small perturbation of $\sigma$ in the form

$$
\sigma=-\frac{1+d}{2}-\frac{\varepsilon_{2}}{2}(p+i q)
$$

Here $\varepsilon_{2}$ is a small parameter, $p$ and $q$ are constants. After some calculations we find the critical value of the Zeldovich number:

$$
Z=Z_{0}+\frac{a_{0}\left(2 a_{0}^{3}-a_{0}^{2}-8 a_{0}+5\right)}{4\left(a_{0}-1\right)^{4}} p \varepsilon_{2}+\frac{a_{0}\left(a_{0}^{2}+2 a_{0}-5\right)}{4\left(a_{0}-1\right)^{4}} q \varepsilon_{2}+o\left(\varepsilon_{2}^{2}\right)
$$

where $Z_{0}$ is a critical value of the Zeldovich number for $\varepsilon_{2}=0$,

$$
Z_{0}=\frac{a_{0}^{2}+3 a_{0}-2}{a_{0}-1},
$$

$a_{0}=1+\sqrt{1+4 k^{2} / u^{2}}$.

Since the coefficients $p$ and $q$ in (6.15) are positive $\left(a_{0} \geq 2\right)$, we can conclude, in particular, that increase of the absolute value of the real and of the imaginary part of $\sigma$ makes the front more stable.

7. Form of the perturbations. We exclude the pressure $p$ and the components $v_{x}$ and $v_{y}$ of the velocity from the system (5.2)-(5.4). To do this we use the transformation rot rot and take the projection of the obtained system on the $z$-axis.

Thus we can rewrite $(5.2),(5.3)$ in the form

$$
\begin{gathered}
\frac{\partial \tilde{\theta}}{\partial t}-u \frac{\partial \tilde{\theta}}{\partial z_{2}}+\tilde{v}_{z} \theta_{s}^{\prime}=\Delta \tilde{\theta} \\
\frac{\partial}{\partial t} \Delta \tilde{v}_{z}-u \frac{\partial}{\partial z_{2}} \Delta \tilde{v}_{z}=P \Delta \Delta \tilde{v}_{z}+Q \Delta_{1} \tilde{\theta}
\end{gathered}
$$

where

$$
\Delta_{1}=\frac{\partial^{2}}{\partial x^{2}}+\frac{\partial^{2}}{\partial y^{2}}
$$


Substituting (6.1), (6.2) into (7.1), (7.2), we obtain

$$
\begin{gathered}
\omega\left(v^{\prime \prime}-k^{2} v\right)-u\left(v^{\prime \prime \prime}-k^{2} v^{\prime}\right)=P\left(v^{\prime \prime \prime \prime}-2 k^{2} v^{\prime \prime}+k^{4} v\right)-Q k^{2} \theta \\
\omega \theta-u \theta^{\prime}+v \theta_{s}^{\prime}=\theta^{\prime \prime}-k^{2} \theta
\end{gathered}
$$

(the index 2 is omitted). Introducing the operators

$$
\begin{aligned}
& L_{1} v=P v^{\prime \prime \prime \prime}+u v^{\prime \prime \prime}-\left(2 P k^{2}+\omega\right) v^{\prime \prime}-u k^{2} v^{\prime}+k^{2}\left(P k^{2}+\omega\right) v, \\
& L_{2} \theta=\theta^{\prime \prime}+u \theta^{\prime}-\left(k^{2}+\omega\right) \theta
\end{aligned}
$$

we can rewrite $(7.3),(7.4)$ in the form

$$
L_{1} v=Q k^{2} \theta, \quad L_{2} \theta=-u e^{-u z_{2}} v
$$

Remember that we consider perturbations decaying at infinity, $v(\infty)=0, \theta(\infty)=0$.

The general solution of (7.5) has the form

$$
\begin{aligned}
& v\left(z_{2}\right)=b_{1} w_{1}\left(z_{2}\right)+b_{2} w_{2}\left(z_{2}\right)+b_{3} w_{3}\left(z_{2}\right), \\
& \theta\left(z_{2}\right)=b_{1} s_{1}\left(z_{2}\right)+b_{2} s_{2}\left(z_{2}\right)+b_{3} s_{3}\left(z_{2}\right) .
\end{aligned}
$$

Here $b_{1}, b_{2}, b_{3}$ are arbitrary constants,

$$
\begin{gathered}
w_{i}=\sum_{j=1}^{\infty} a_{i j} e^{\sigma_{i j} z_{2}}, \quad s_{i}=\sum_{j=1}^{\infty} c_{i j} e^{\sigma_{i j} z_{2}}, \quad i=1,2,3, \\
\sigma_{11}=-\frac{u}{2}-\sqrt{\frac{u^{2}}{4}+k^{2}+\omega}, \quad \sigma_{21}=-k, \quad \sigma_{31}=-\frac{u}{2 P}-\sqrt{\frac{u^{2}}{4 P^{2}}+k^{2}+\frac{\omega}{P}},
\end{gathered}
$$

$k$ is supposed, for certainty, to be positive,

$$
\begin{gathered}
\sigma_{i j}=\sigma_{i, j-i}-u, \quad i=1,2,3, j=2,3, \ldots, \\
c_{11}=1, \quad c_{21}=c_{31}=0, \quad a_{21}=a_{31}=1, \\
a_{1 j}=\frac{Q k^{2} c_{1 j}}{\left(\sigma_{1 j}^{2}-k^{2}\right)\left(P \sigma_{1 j}^{2}+u \sigma_{1 j}-\left(P k^{2}+\omega\right)\right)}, \quad j=1,2,3, \ldots, \\
c_{i, j+1}=\frac{-u a_{i j}}{\sigma_{i, j+1}^{2}+u \sigma_{i, j+1}-\left(k^{2}+\omega\right)}, \quad i=1,2,3, j=1,2,3, \ldots, \\
a_{i, j+1}=\frac{Q k^{2} c_{i, j+1}}{\left(\sigma_{i, j+1}^{2}-k^{2}\right)\left(P \sigma_{i, j+1}^{2}+u \sigma_{i, j+1}-\left(P k^{2}+\omega\right)\right)}, \quad i=2,3, j=1,2,3, \ldots
\end{gathered}
$$


We assume here that

$$
\left(\sigma_{1 j}^{2}-k^{2}\right)\left(P \sigma_{1 j}^{2}+u \sigma_{1 j}-\left(P k^{2}+\omega\right)\right) \neq 0
$$

for $i=1, j=1,2,3, \ldots$ and $i=2,3, j=2,3, \ldots$,

$$
\sigma_{i j}^{2}+u \sigma_{i j}-\left(k^{2}+\omega\right) \neq 0
$$

for $i=1,2,3, j=2,3, \ldots$.

It is easy to verify that the series (7.6) converge, and the solutions $\left(w_{i}, s_{i}\right), i=1,2,3$, are linearly independent if the numbers $\sigma_{i 1}$ are different. The last condition means, in particular, that $P \neq 1$ and $P \neq \infty$.

We show now that the functions $\left(w_{i}, s_{i}\right)$ satisfy (7.5). Indeed, we denote

$$
w_{i}^{n}=\sum_{j=1}^{n} a_{i j} e^{\sigma_{i j} z_{2}}, \quad s_{i}^{n}=\sum_{j=1}^{n} c_{i j} e^{\sigma_{i j} z_{2}}, \quad i=1,2,3 .
$$

Substituting these partial sums into (7.5), we have

$$
L_{1} w_{i}^{(n)}=Q k^{2} s_{i}^{(n)}, \quad L_{2} s_{i}^{(n)}=-u e^{-u z_{2}}\left(w_{i}^{(n)}-a_{i n} e^{\sigma_{i n} z_{2}}\right)
$$

Passing to the limit as $n \rightarrow \infty$, we obtain that (7.6) satisfies (7.5).

We determine now the function $\sigma(R, P, k, \omega)$ (see (6.11)). From the boundary conditions for the velocity

$$
\sum_{i=1}^{3} b_{i} w_{i}(0)=0, \quad \sum_{i=1}^{3} b_{i} w_{i}^{\prime}(0)=0
$$

Thus

$$
\sigma=\frac{1}{u} \frac{\frac{b_{1}}{b_{3}} s_{1}^{\prime}(0)+\frac{b_{2}}{b_{3}} s_{2}^{\prime}(0)+s_{3}^{\prime}(0)}{\frac{b_{3}}{b_{3}} s_{1}(0)+\frac{b_{2}}{b_{3}} s_{2}(0)+s_{3}(0)}
$$

where

$$
\begin{aligned}
& \frac{b_{1}}{b_{3}}=-\frac{w_{3}(0) w_{2}^{\prime}(0)-w_{3}^{\prime}(0) w_{2}(0)}{w_{1}(0) w_{2}^{\prime}(0)-w_{1}^{\prime}(0) w_{2}(0)} \\
& \frac{b_{2}}{b_{3}}=\frac{w_{3}(0) w_{1}^{\prime}(0)-w_{3}^{\prime}(0) w_{1}(0)}{w_{1}(0) w_{2}^{\prime}(0)-w_{1}^{\prime}(0) w_{2}(0)}
\end{aligned}
$$

8. Stability conditions. Though we have an explicit formula for the function $\sigma$, the dispersion relation (6.10) cannot be studied analytically in the general case. In this section we present the results of its numerical analysis and consider analytically the case 
of small Rayleigh numbers. We obtain from (7.8):

$$
\sigma=-\frac{1+d}{2}-\frac{4 P^{2} R}{u^{3}} \frac{\tilde{k}^{2}}{(1+d)(1+d+\tilde{k})^{2}(1+\tilde{d}+P(1+d))(1-\tilde{d}-P(1+d))}+o(R)
$$

where

$$
d=\sqrt{1+\tilde{k}^{2}+4 \omega_{1}}, \quad \tilde{d}=\sqrt{1+\tilde{k}^{2} P^{2}+4 \omega_{1} P}, \quad \tilde{k}=\frac{2 k}{u}, \quad \omega_{1}=\frac{\omega}{u^{2}}
$$

To find the cellular stability boundary we put $\omega=0$. We see from (8.1) that for $R$ positive, which correspond to ascending fronts, $\sigma$ increases in comparison with the case $R=0$, and the equation $\sigma=-1$, which determines the stability boundary, can have a solution for $k>0$. For $R$ negative, which correspond to descending fronts, $\sigma$ decreases.

Figure 1 shows $\sigma$ as a function of $k$ for different values of the Rayleigh number, found

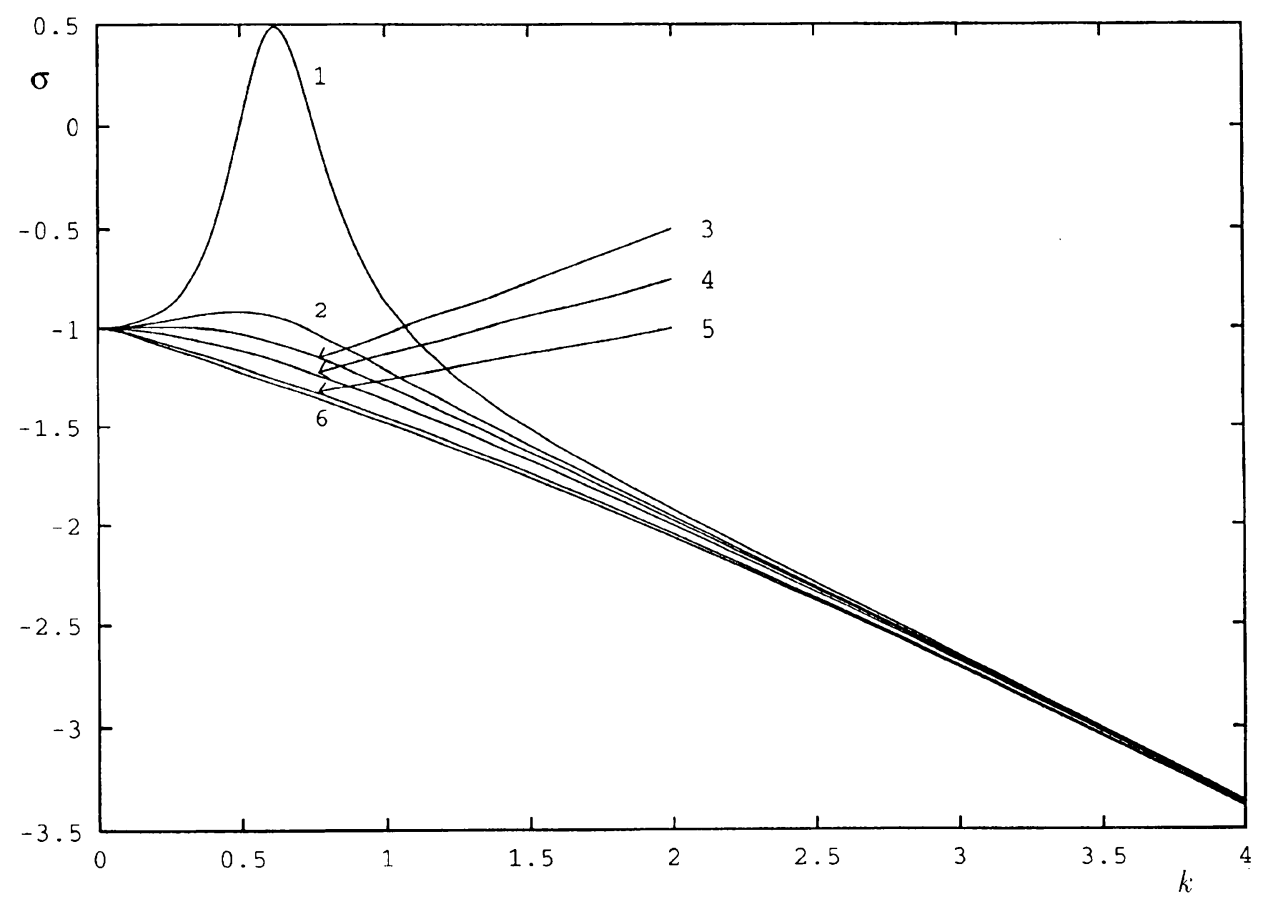

FIG. 1. $\sigma$ as a function of wavenumber for different $R . P=0.99$, $u=\sqrt{2}, 1 . R=50,2 . R=30,3 . R=18,4 . R=0,5 . R=-40,6$. $R=-60$. 


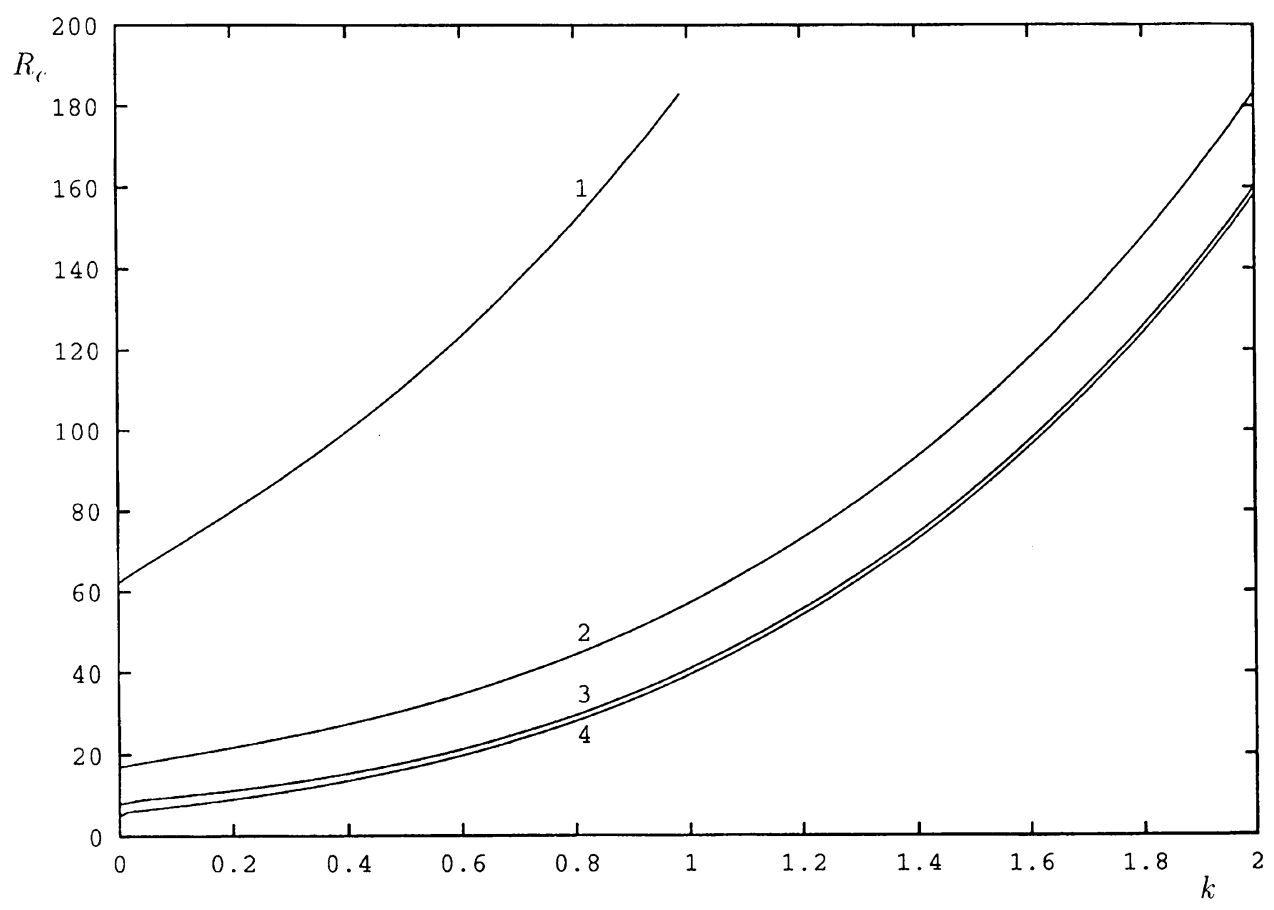

FIG. 2. Critical values of the Rayleigh number as a function of wavenumber for different values of the Prandtl number. $u=\sqrt{2}, 1$. $P=0.1,2 . P=0.5,3$. $P=2,4 . P=30$.

from (7.8). We see that for $R$ positive and sufficiently large, $\sigma$ becomes more than -1 , i.e., the instability occurs. For $\sigma$ negative it does not take place.

If we put $\sigma=-1$ in equation (8.1) we find the critical value of the Rayleigh number:

$$
R_{c}=\frac{u^{3}}{16 P^{2}}(1+d)(1+d+\tilde{k})^{2}[1+\tilde{d}+P(1+d)][-1+\tilde{d}+P(1+d)]
$$

This approximate formula shows that $R_{C}$ is an increasing function of wavenumber with its minimal value at $k=0$. We cannot expect that (8.2) gives a good approximation of the critical value of the Rayleigh number for all values of the parameters. However, it reflects qualitatively its dependence on the parameters. Figure 2 shows the critical value of the Rayleigh number as a function of wavenumber for different values of the Prandtl number, found numerically as a solution of (6.12), (7.8). In Fig. 3 (see p. 240) this dependence is shown for different values of the front velocity. We conclude that increase of the front velocity makes the front more stable, while increase of the Prandtl number destabilizes it. 


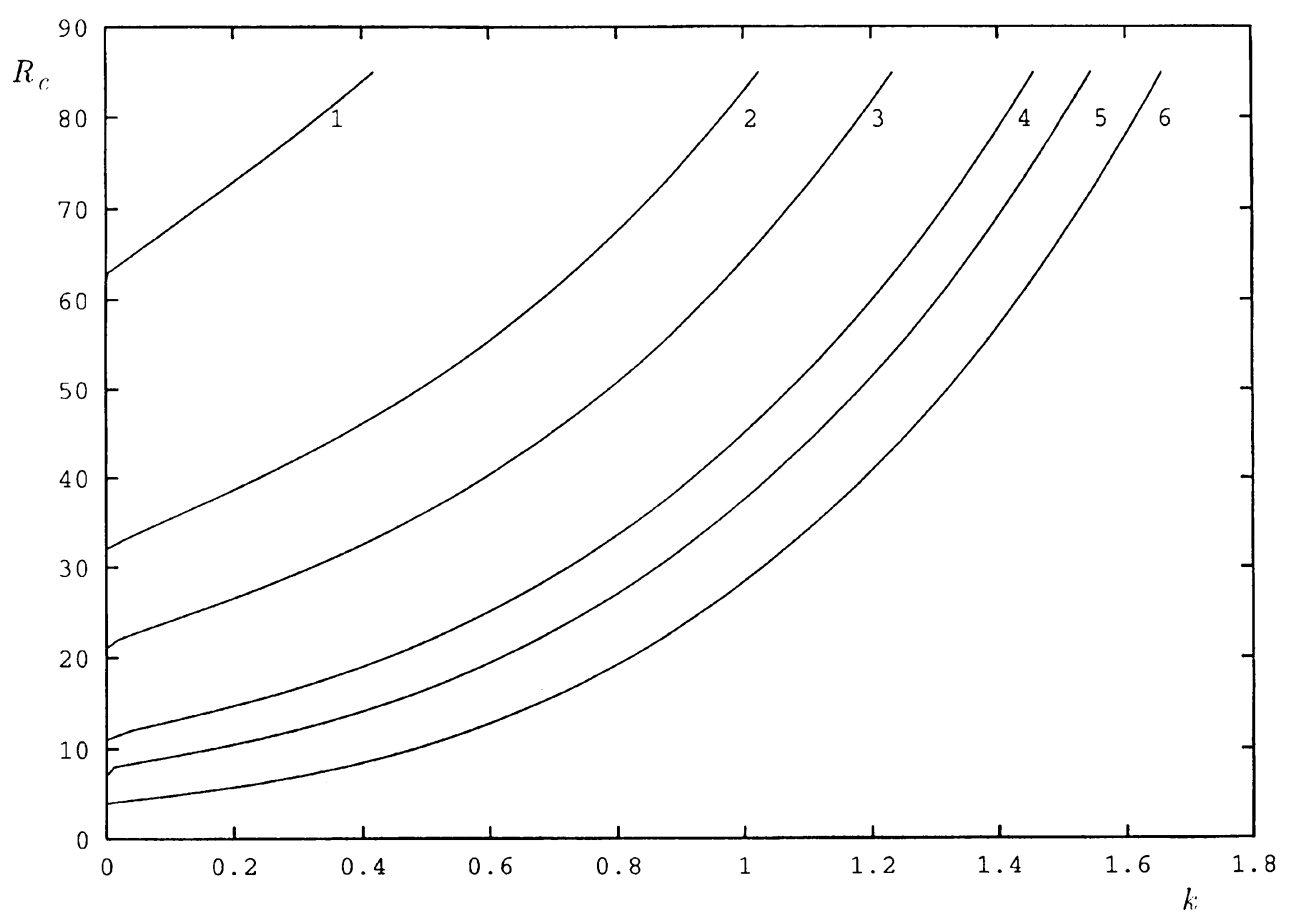

FIG. 3. Critical values of the Rayleigh number as a function of wavenumber for different values of the front velocity. $P=0.99,1$. $u=1.0,2 . u=1.25,3 . u=\sqrt{2}, 4 . u=1.75,5 . u=2,6 . u=2.5$.

The comparison of $R_{C}$ found from (8.2) and (6.12), (7.8) is given in Fig. 4. We see that (8.2) gives a good approximation for small wavenumbers. We have from it in the limit as $k \rightarrow 0$ :

$$
R_{c}=\frac{2 u^{3}(1+P)}{P}
$$

To find an oscillatory stability boundary we put $\omega_{1}=i \phi_{1}$. For small $R$ from (8.1) we obtain (6.14) with

$$
\varepsilon_{2}=\frac{2 P^{2} R}{u^{3}}, \quad p=\frac{\tilde{k}^{2} S_{1}}{S_{1}^{2}+S_{2}^{2}}, \quad q=\frac{\tilde{k}^{2} S_{2}}{S_{1}^{2}+S_{2}^{2}}
$$

where

$$
S=T_{1} T_{3}-T_{2} T_{4}, \quad S_{2}=-\left(T_{1} T_{4}+T_{2} T_{3}\right)
$$




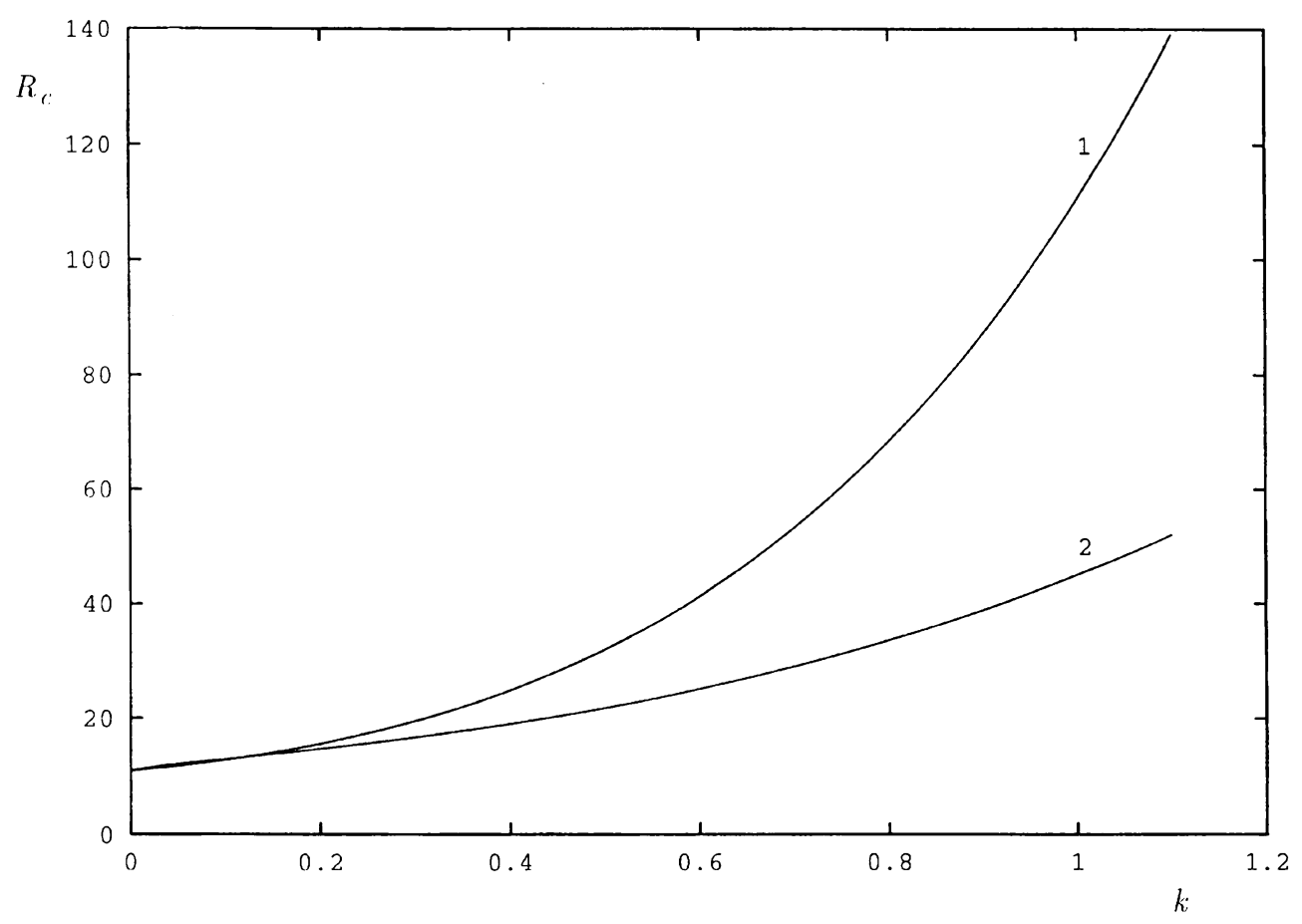

Fici. t. Comparison of $R_{c}$ found from (8.2) (1.) and (6.12), (7.8) (2.).

$$
\begin{aligned}
& T_{1}=(1+a)\left((1+a+\tilde{k})^{2}-b^{2}\right)-2 b^{2}(1+a+\tilde{k}), \\
& T_{2}=b\left(2(1+a)(1+a+\tilde{k})+(1+a+\tilde{k})^{2}-b^{2}\right), \\
& T_{3}=1-(\tilde{a}+P+a P)^{2}+(\tilde{b}+b P)^{2} \\
& T_{1}=-2(\tilde{a}+P+a P)(\tilde{b}+b P), \\
& \quad a+i b=d, \quad \tilde{a}+i \tilde{b}=\tilde{d} .
\end{aligned}
$$

The constants $p$ and $q$ for $a=a_{0}$ and $P=1$, taken as an example, are found to be positive; so the hydrodynamical perturbations make the front more stable for $R$ small and positive, and more unstable for $R$ small and negative. Negative $R$ correspond to descending fronts.

If $R$ is not small the dispersion relation can be analyzed numerically. Figure 5 (see p. 242 ) shows the critical values of the Zeldovich number as a function of wavenumber for different values of the Rayleigh number. Here $\sigma$ is taken in the form (7.8). 


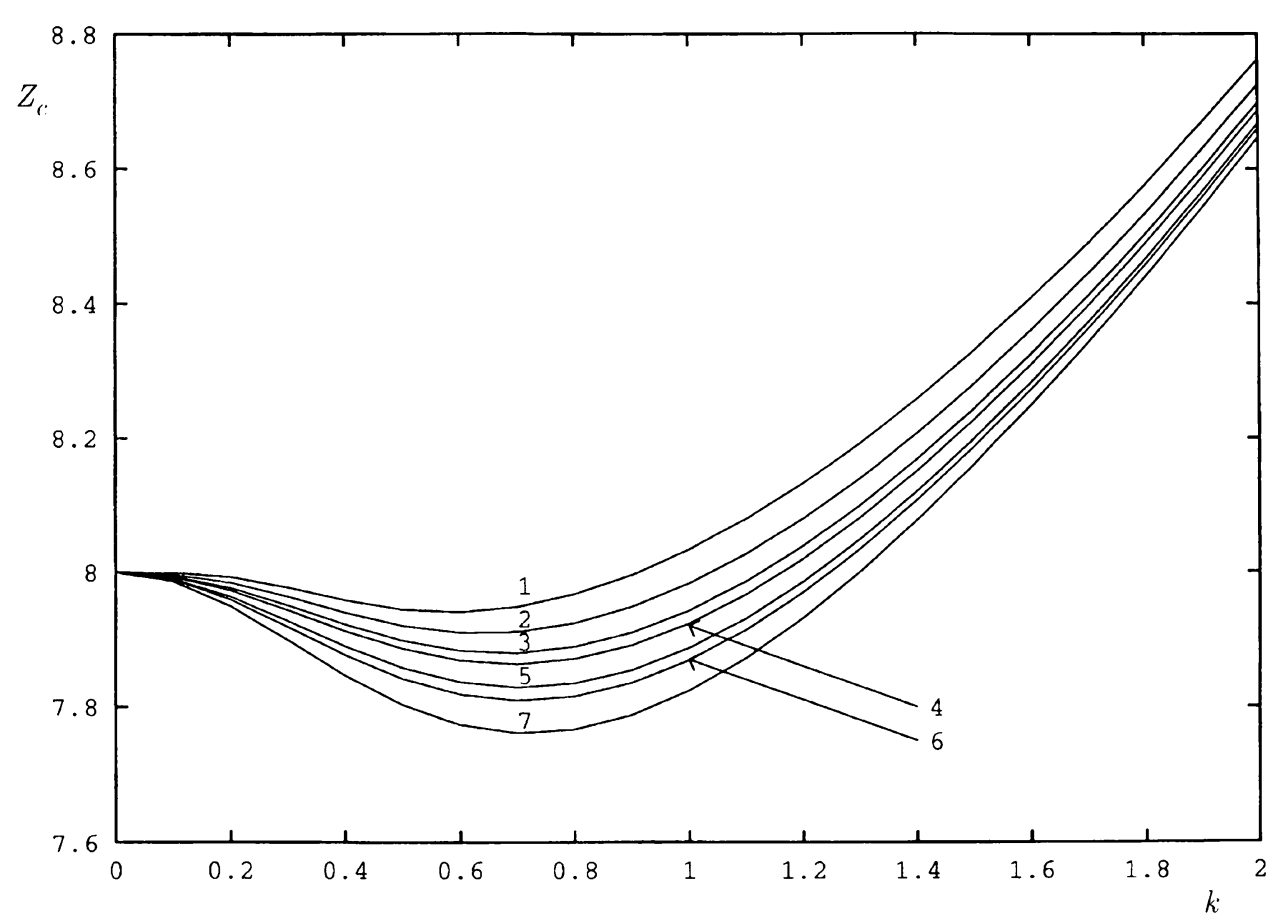

FIG. 5. Critical values of the Zeldovich number as a function of wavenumber for different values of the Rayleigh number. $P=0.5$, $u=\sqrt{2}, 1 . R=150,2$. $R=100,3 . R=60,4$. $R=40,5 . R=0,6$. $R=-20,7 . R=-60$.

Figure 6 shows qualitatively the stability boundaries on the $(R, Z)$-plane. The oscillatory instability boundary is independent of $R$ for $k=0$ and practically independent for $k \geq 2$. The stability region is below the curve that determines the boundary. The cellular instability boundary is always independent of $Z$. The stability region is to the left of the stability boundary. 


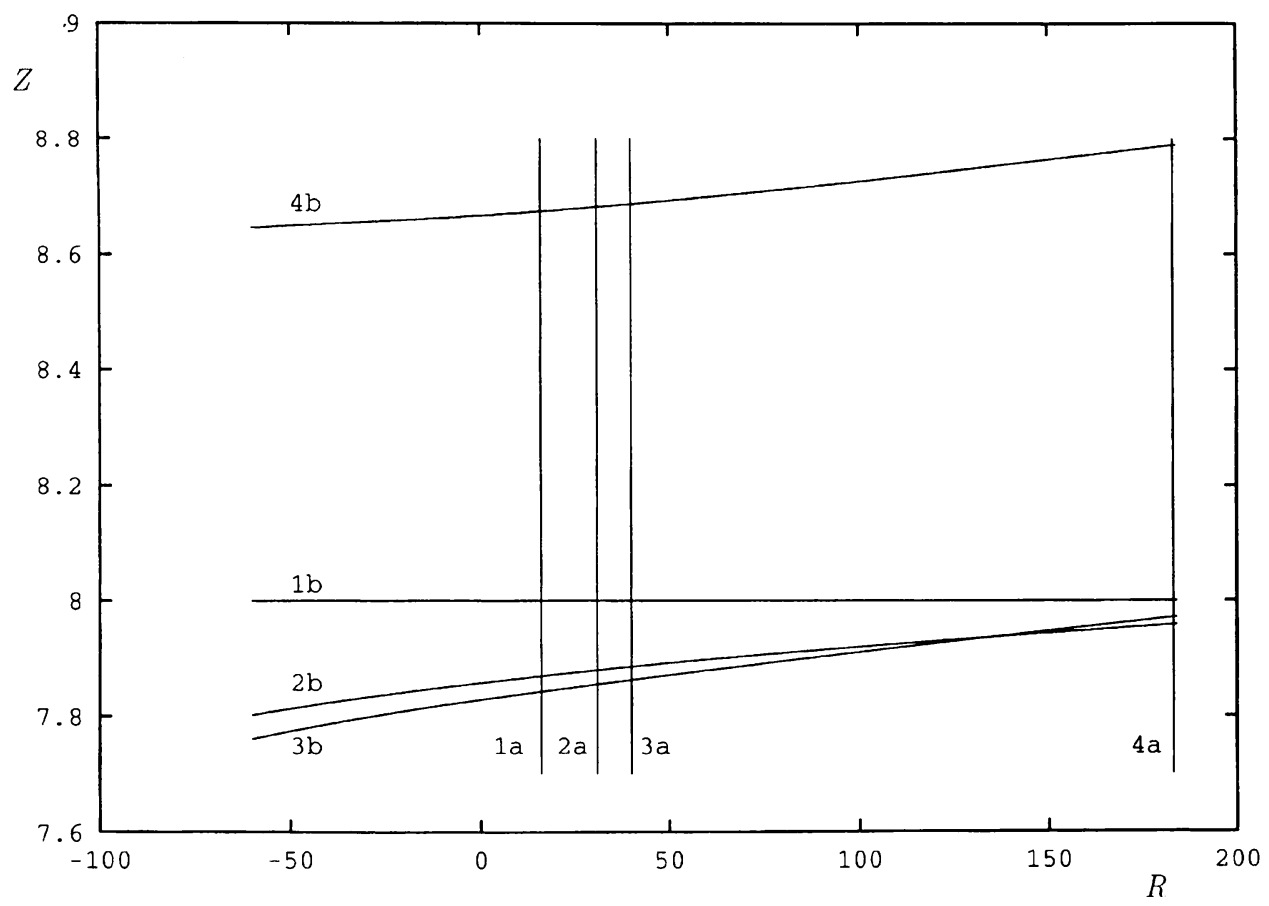

FIG. 6. Stability regions on the $(R, Z)$-plane for different values of the wavenumber. (1a) $(\omega=0, k=0)$ (1b) $(\omega=i \phi, k=0)$ (2a) $(\omega=0, k=0.5)(2 \mathrm{~b})(\omega=i \phi, k=0.5)(3 \mathrm{a})(\omega=0, k=0.7)(3 \mathrm{~b})$ $(\omega=i \phi, k=0.7)(4 \mathrm{a})(\omega=0, k=2)(4 \mathrm{~b})(\omega=i \phi, k=2)$

9. Discussion. We have studied the linear stability of exothermic reaction fronts with original reactants considered as a homogeneous incompressible liquid and a solid product. Basic parameters that determine the front stability are the Rayleigh number and the Zeldovich number. Both cellular instability and oscillatory instability can occur.

The cellular instability boundary corresponds to the case when the eigenvalue with the maximal real part intersects the imaginary axis through zero. The cellular instability occurs for positive and sufficiently large values of the Rayleigh number. The conditicn of cellular instability is independent of the Zeldovich number. It is interesting to note that there is no perturbation of temperature behind the reaction zone in this case. The temperature is constant and equal to the burning temperature. So this case can be considered as a pure convective instability. Increase of the front velocity makes it more stable. For negative values of the Rayleigh number there is no instability.

We now give a physical interpretation of the results concerning cellular instability. For the ascending fronts, which correspond to positive values of the Rayleigh number, the exothermic chemical reaction can cause convective instability because of the temperature gradient in front of the reaction zone. It is similar to some extent to the classical RayleighBenard problem of convection in a layer of a liquid heated from below. If the liquid is heated from above then, as is well known, cellular instability does not occur. This is the same in the problem under consideration for the descending fronts (negative $R$ ). 
The influence of the front velocity on the stability condition also has a clear physical explanation. In the moving coordinate frame where the reaction zone is unmovable, there is a flow of the liquid towards the front. The convective instability leads to the liquid motion in the direction opposite to the direction of the flow. So the greater the flow velocity the more it is possible that the perturbations decay.

The oscillatory instability boundary corresponds to the case when a pair of complex conjugate eigenvalues with nonzero imaginary parts intersects the imaginary axis. Similar to condensed phase combustion the instability takes place for large values of the Zeldovich number. The stability conditions also depend here on the direction of the front propagation.

The front propagating upward appears to be more stable with respect to multidimensional perturbations $(k \neq 0)$ than in the pure combustion problem without hydrodynamics. The physical explanation for this can be as follows. For the condensed phase combustion the instability of the front leads to the appearance of high-temperature heads which move in front of the reaction zone. If the reactants are in a liquid phase then appearance of a high-temperature head causes the liquid motion, which in its turn decreases the temperature of the head, and the perturbation can decay.

For a front propagating downward the influence of liquid motion is opposite. Convection of the liquid decreases the heat loss from a high-temperature head to the unreacted reactants caused by heat diffusion. The heat is conserved near the reaction front, and the perturbation of the temperature has better conditions to increase.

The last of our remarks concerns the method of the analysis. It is a well-known method based on the approximation of an infinitely narrow reaction zone. This means that we neglect the reaction term outside the reaction zone and replace it by the jump conditions in the reaction zone. The dispersion relation is derived as a result of linearization of the jump conditions. The connection of the dispersion relation with the external problem exists due to the dimensionless temperature gradient $\sigma=\theta^{\prime} /(u \theta)$ that appears in the former and should be found from the latter. However, some of the conclusions can be made without its explicit form. In particular, as was mentioned above, the cellular instability condition is independent of the Zeldovich number and is given by the equality $\sigma=-1$. The conclusion that there is no perturbation of temperature behind the reaction zone is also general for this kind of jump conditions. One more general conclusion is that increase of the absolute values of the real and imaginary parts of $\sigma$, i.e., the temperature gradient, makes the reaction front more stable, while decrease of them destabilizes the front.

\section{REFERENCES}

[1] A. P. Aldushin and S. G. Kasparyan, Thermo-diffusion instability of combustion front. Doklady Akademii Nauk SSSR (5) 244, 67 70 (1979) (Russiau)

[2] A. S. Babadzhanyan, Vit. A. Volpert, V1. A. Volpert, S. P. Dartyan, and I. N. Megrabora. Frontal regimes of an exothermal reaction with radially symmetric injection of reagents. Combustion. Explosion and Shock Waves (6) 24, 711 719 (1988)

[3] A. S. Babadzhanyan, Vit. A. Volpert, Vl. A. Volpert, S. P. Davtyan, and I. N. Megrabova, Stability of frontal regimes for the occurrence of an exothermic reaction with radially symmetric supply of the reagents. Combustion. Explosion and Shock Waves (1) 25. 2:3-31 (1989) 
[4] G. I. Barenblatt, Ya. B. Zeldovich, and A. G. Istratov, Diffusive-thermal stability of a laminar flame, Zh. Prikl. Mekh. Tekh. Fiz. (4) 21 (1962) (Russian)

[5] A. Bayliss and B. J. Matkowsky, Nonlinear dynamics of cellular flames, SIAM J. Applied Math. (2) 52, 396-415 (1992)

[6] G. Bazsa and I. R. Epstein, Traveling waves in the nitric acid-iron (II) reaction, J. Phys. Chem. (14) 89, 3050-3053 (1985)

[7] V. P. Begisher. V. A. Volpert, S. P. Davtyan, and A. Y. Malkin, Some features of the anionic activated $\varepsilon$-caprolactam polymerization under wave propagation conditions, Dokl. Phys. Chemistry (4) 29, $1075 \cdot 1077(1984)$

[8] S. A. Bostandzhiyan, V. I. Boyarchenko, P. V. Zhirkov, and Zh. A. Zinenko, Lou-temperature polymerization conditions in a flow-through reactor, J. Appl. Mech. Tech. Phys. (1) 20, 99-104 (1979)

[9] S. A. Bostandzhiyan, M. V. Shulikovskaya, and S. P. Davtyan, 6th International School on Modeling Heat and Mass Transfer Processes in Chemical and Biochemical Reactors, Varna, 1989, p. 231

[10] A. A. Butakov, E. I. Maksimov, and K. G. Shkadinsky, Theory of chemical displacement reactors, Combustion, Explosion and Shock Waves (1) 14, 48-54 (1978)

[11]. A. A. Butakor and E. A. Stessel, Influence of convection on occurrence of the exothermic reaction in tubular reactors, Doklady Akademii Nauk SSSR (6) 237, 1422-1425 (1977) (Russian)

[12] A. A. Butakov and A. M. Zanin, Effects of viscosity variation on an exothermic reaction in a flow system, Combustion, Explosion and Shock Waves (5) 14, 628 631 (1978)

[13] N. M. Chechilo, R. Ya. Khvilivitsky, and N. S. Enikolopyan, The phenomenon of propagation of the polymerization reaction, Dokl. Phys. Chemistry (4-6) 204, 512-513 (1972)

[14] N. M. Chechilo and N. S. Enikolopyan, Structure of the polymerization wave front and propagation mechanism of the polymerization reaction, Dokl. Phys. Chemistry (4-6) 214, 174-176 (1974)

[15] N. M. Chechilo and N. S. Enikolopyan, Effect of the concentration and nature of the initiators on the propagation process in polymerization, Dokl. Phys. Chemistry (4-6) 221, $391-394$ (1975)

[16] N. M. Chechilo and N. S. Enikolopyan, Effect of pressure and initial temperature of the reaction mixture during propagation of a polymerization reaction, Dokl. Phys. Chemistry (1 3) 230, 840 843 (1976)

[17] S. P. Davtyan, P. V. Zhirkov, and S. A. Vol'fson, Problems of non-isothermal character in polymerization processes, Russ. Chemical Reviews (2) 53, 150-163 (1984)

[18] D. A. Frank-Kamenetskii, Diffusion and Heat Transfer in Chemical Kinetics, Plenum Press, New York, 1969

[19] M. Garbey, H. G. Kaper, G. K. Leaf, and B. J. Matkowsky, Nonlinear analysis of condensed phase surface combustion, European J. Applied Math. 1, 73-89 (1990)

[20] M. Garbey, H. G. Kaper, G. K. Leaf, and B. J. Matkowsky, Linear stability analysis of cylindrical flames, Quart. Appl. Nath. XLVII, 691-704 (1989)

[21] M. Garbey, H. G. Kaper, G. K. Leaf, and B. J. Matkowsky, Using Maple for the analysis of bifurcation phenomena in condensed phase surface combustion, J. Symbolic Computation 12, 89-113 (1991)

[22] G. Z. Gershuni and E. M. Zhuhovitsky, Convective Instabilities of Incompressible Liquid, Moscow, Nauka. 1972

[23] S. I. Khudyaev, On the asymptotic theory of the stationary combustion wave, Khimicheskaya Physica (5) 6, 681-691 (1987) (Russian)

[24] A. Ya. Malkin, Yu. B. Navochnik, and V. P. Begishev, Polymer Process Engrg. 1, 71 (1983)

[25] S. B. Margolis, An asymptotic theory of condensed two-phase flame propagation, SIAM J. Appl. Math. 43, 351-369 (1983)

[26] S. B. Margolis and R. C. Armstrong, Two asymptotic models for solid propellant combustion, Combust. Sci. Technol. 47, 1-38 (1986)

[27] S. B. Margolis, H. G. Kaper, G. K. Leaf, and B. J. Matkowsky, Bifurcation of pulsating and spinning reaction fronts in condensed two-phase combustion, Combustion Sci. and Tech. 43, 127 165 (1985)

[28] B. J. Matkowsky and G. I. Sivashinsky, Propagation of a pulsating front in solid fuel combustion, SIAM J. Appl. Math. 35, 465-478 (1978)

[29] A. G. Merzhanov, SHS-process: Combustion theory and practice, Archivum Combustionis (1-2) 1, $2348(1981)$ 
[30] A. G. Merzhanov, A. K. Filonenko, and I. P. Borovinskaya, New phenomena in combustion of condensed systems, Dokl. Phys. Chem. 208, 122125 (1973)

[31] A. G. Merzhanov and B. I. Khaikin, Theory of combustion waves in homogeneous media, Progr. Ener. Combustion Sci. 14, 1-98 (1988)

[32] H. Miike, S. C. Muller, and B. Hess, Oscillatory deformation of chemical waves induced by surface flow, Physical Review Letters (18) 61, 2109-2112 (1988)

[33] H. Miike, S. C. Muller, and B. Hess, Oscillatory hydrodynamic flow induced by chemical waves, Chem. Physics Letters $(5,6) \mathbf{1 4 4}, 515-520(1988)$

[34] I. Nagypal, G. Bazsa, and I. R. Epstein, Gravity-induced anisotropies in chemical waves, J. Amer. Chem. Soc. (13) 108, 3635 -3640 (1986)

[35] B. V. Novozhilov, The rate of propagation of the front of an exothermic reaction in a condensed phase, Proc. Academy Sci. USSR, Phys. Chem. Sect. 141, 836838 (1961)

[36] T. Plesser, H. Wilke, and K. H. Winters, Interaction of chemical waves with convective flows induced by density gradients. A comparison between experiments and computer simulations, Chem. Physics Letters $(1,2)$ 200, 158-162 (1992)

[37] J. A. Pojman, R. Graven, A. Khan, and W. West, Convective instabilities in traveling fronts of addition polymerization, J. Physical Chemistry 96, 7466-7472 (1992)

[38] J. A. Pojman and I. R. Epstein, Convective effects on chemical waves. 1. Mechanisms and stability criteria, J. Phys. Chem. (12) 94, 4966-4972 (1990)

[39] J. A. Pojman, I. R. Epstein, T. J. McManus, and K. Scowalter, Convective effects on chemical waves. 2. Simple convection in the iodate-arsenous acid system. J. Phys. Chem. (3) 95, 12991306 (1991)

[40] J. A. Pojman, I. P. Nagy, and I. R. Epstein, Convective effects in chemical waves. 3. Multicomponent convection in the iron (II)-nitric acid system, J. Phys. Chem. (3) 95, 1306-1311 (1991)

[41] D. S. Riley and S. H. Davis, Long-wave interactions in morphological and convective instabilities, IMA J. Applied Math. 45, 267-285 (1990)

[42] S. E. Solovyov, V. A. Volpert, and S. P. Davtyan, Radially symmetric flow of reacting liquid with changing viscosity, SIAM J. Appl. Math. 53, 907-914 (1993)

[43] M. V. Shulikovskaya, S. A. Bostandzhiyan, and S. P. Davtyan, Frontal radical polymerization in spherical plug-flow reactors, Teor. osnovy khim. tehnologii (3) 23, 340-345 (1989) (Russian)

[44] A. G. Strunina, A. V. Dvoryankin, and A. G. Merzhanov, Unstable regimes of thermite system combustion, Combustion, Explosion and Shock Waves 19, 158-163 (1983)

[45] D. A. Vaganov, Zh. Prikl. Mekh. Tekh. Fiz. (1), 114-122 (1977) (Russian)

[46] D. A. Vaganov and S. I. Khudyaev, On a stationary problem of combustion theory, Combustion, Explosion and Shock Waves 5, 167-172 (1969)

[47] D. A. Vasquez, J. W. Wilder, and B. F. Edwards, Hydrodynamic instability of chemical waves, J. Chem. Phys. (3) 98, 2138.2143 (1993)

[48] D. A. Vasquez, B. F. Edwards, and J. W. Wilder. Onset of convection for autocatalytic reaction fronts: Laterally bounded systems, Physical Review A 43, 66946699 (1991)

[49] H. J. Viljoen, J. E. Gatica, and V. Hlavacek, Bifurcation analysis of chemically driven convection. Chem. Engrg. Sci. (2) 45, 503-517 (1990)

[50] Vit. A. Volpert, Vl. A. Volpert, S. P. Davtyan, I. N. Megrabova, and N. F. Surkov, Two-dimensional combustion modes in condensed flow, SIAMI J. Appl. Math. 52. $368 \cdot 383$ (1992)

[51] V. A. Volpert, A. I. Volpert, and A. G. Merzhanov, Application of bifurcation theory to investigation of spin waves of combustion, Dokl. Phys. C'hem. 262, 55-58 (1982)

[52] V. A. Volpert, A. I. Volpert, and A. G. Merzhanov, Analysis of nonunidimensional combustion regimes by bifurcation theory methods, Dokl. Phys. Chem. 263, 239-242 (1982)

[53] V. A. Volpert and S. P. Davtyan, The problem of wave existence for the process of polymerization and crystallization, Dokl. Akademii Nauk SSSR (1) 268. 62 65 (1983)

[54] V. A. Volpert, I. N. Megrabova, S. P. Davtyan, and V. P. Begishev, Propagation of caprolactam polymerization wave, Combustion, Explosion and Shock Waves (4) 21, 441-447 (1985)

[55] Vit. Volpert and Vl. Volpert, Propagation of frontal polymerization-crystallization waves, European J. Appl. Math. 5, 201215 (1994)

[56] Vit. A. Volpert and Vl. A. Volpert, Propagation velocity estimation for condensed phase combustion, SIAM J. Applied Math. (4) 51, 10741089 (1991)

[57] Ya. B. Zeldovich, G. I. Barenblatt, V. B. Librovich, and G. M. Makhviladze, The Mathematical Theory of Combustion and Explosions, Consultants Bureau, New York, 1985 
[58] Ya. B. Zeldovich and D. A. Frank-Kamenetsky, The theory of thermal propagation of flames, Zh. Fiz. Khim. 12, 100-105 (1938) (Russian)

[59] G. V. Zhizhin and A. S. Segal, Hydrodynamic stability of the spherical front of a reaction accompanied by a strong increase in viscosity, Fluid Dynamics (3) 23, 361-367 (1988)

[60] G. V. Zhizhin and A. S. Segal, Hydrodynamic stability of a cylindrical reaction front associated with a strong increase of viscosity, J. Appl. Mech. Tech. Phys. (2) 29, 216-224 (1988) 